\title{
Sequence-based protein kinase inhibition: applications for drug development
}

Isaiah D. Wexler ${ }^{1}$, Masha Y. Niv², and Hadas Reuveni ${ }^{2}$

${ }^{1}$ Hadassah-Mount Scopus University Hospital, Jerusalem, Israel and ${ }^{2}$ Keryx Biopharmaceuticals, New York, NY, USA

BioTechniques 39:575-576 (October 2005)

doi 10.2144/000112045

Nearly a century ago, Paul Ehrlich proposed his lock and key theory that has remained one of the cornerstones of rational drug development. Disrupting or enhancing the interaction between a ligand and its substrate is the basis for numerous drugs targeting signal transduction processes. Much of drug discovery is based on finding molecular mimics that resemble either the lock or key of a given ligand-substrate pair. The challenge of drug development is the rapid and efficient identification of mimics that are highly selective and specific for the targeted interaction.

Scientists associated with Keryx Biopharmaceuticals (New York, NY, USA) have developed two drug discovery technologies, based on molecular mimicry, that combines rapid development of drug candidates and identification of potential biomarker targets for selected indications. KinAce ${ }^{\mathrm{TM}}$ is a platform technology for the protein-sequence-based development of small peptide-derived kinase inhibitors developed by Shmuel A. Ben-Sasson at the Hebrew University in Jerusalem and Keryx Biopharmaceuticals $(1,2)$. KinScreen ${ }^{\mathrm{TM}}$ is a method for identifying novel target kinases utilizing peptides developed via the KinAce technology. Peptides directed against specific kinases are tested in a series of biomarker assays that serve as in vitro models for a variety of diseases or pathologic processes including cancer, diabetes, angiogenesis, and immune disorders. The advantage of this technique is that it identifies both kinase biomarker targets with potential therapeutic applications and drug candidates suitable for further development (1).

\section{KinAce: TARGETING KINASE-SUBSTRATE INTERACTION SITES}

The rationale behind KinAce is based on the concept that protein kinases have shared structural elements involved in kinasesubstrate interactions and that the same regions can be targeted in multiple kinases, thereby making the platform widely applicable. Despite the overall similarities in structure, there is still sufficient diversity within these segments to allow for the development of selective sequence-based kinase inhibitors. Elements that are involved in substrate-kinase interactions are particularly attractive in that they can serve as templates for the construction of molecular mimics that interfere with kinase-substrate interactions and thereby modulate specific signal transduction pathways.

The initial step in developing the KinAce platform was the identification and characterization of key well-defined regions within protein kinases involved in substrate binding. An example of such a region is the $H J-\alpha G$ region, a 21-amino acid segment located in the catalytic domain of all protein kinases. Within this highly conserved segment are 7-10 amino acids that have been shown, based on crystallographic and mutational analysis, to be involved in substrate binding. Several amino acids in this segment are highly variable among the different protein kinases and thereby provide substrate specificity (Figure 1). Other amino acids in this segment, which are highly conserved and buried within the catalytic domain, serve as a structural scaffold that facilitates presentation of the variable amino acids to specific substrates. From a drug development perspective, the common structural fold and conserved sequence motif of the
$H J-\alpha G$ region among protein kinases facilitates drug design, whereas the amino acid sequence variability of the exposed substrate-binding residues allow for targeting specificity.

\section{SEQUENCE-BASED PEPTIDE INHIBITORS}

The next step in KinAce platform development was the systematic derivation of potential peptide inhibitors based on the sequence of these novel substrate-binding regions. Bioinformatics algorithms were developed, and computational protein modeling was utilized to create peptides 6-14 amino acids in length. To enhance activity and selectivity, the synthesized peptides included amino acid residues that were both variable, highly exposed, and known to be part of the active template involved in substrate binding. To stabilize the structure of these peptides and to reduce polarity, several chemical modifications of the native sequence were introduced. Cell permeability was enhanced by $\mathrm{N}$-myristoylation and $\mathrm{C}$-amidation.

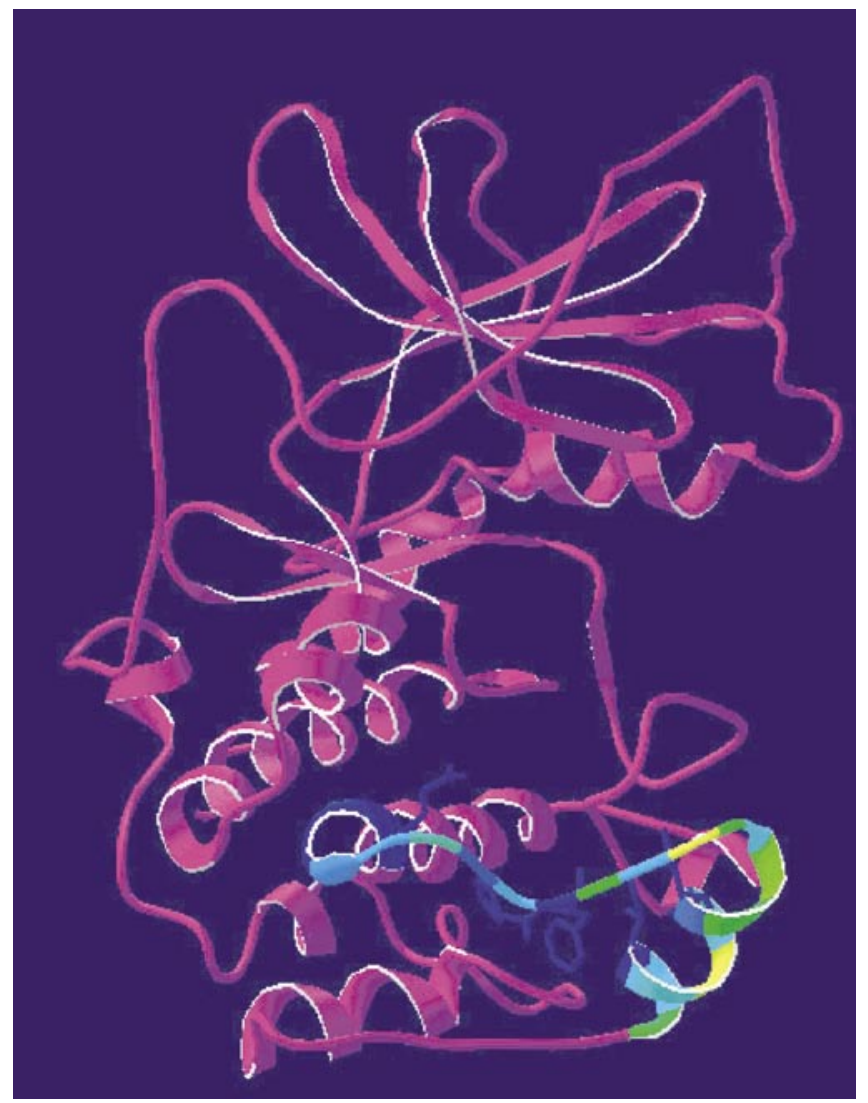

Figure 1. Protein kinase A (PKA; 1BX6.pdb entry in Protein Data Bank) is depicted in ribbon representation. The $\mathrm{HJ}-\alpha \mathrm{G}$ region is colored by accessibility using Swiss-PdbViewer freeware. The colors vary from the buried blue to the accessible yellow. Only the side chains of the conserved residues are shown. These residues are buried in the protein and are spaced by variable, solvent-accessible residues. 
Identification of the best drug candidates was accomplished by screening relatively small libraries of 20-40 peptides that varied in length and amino acid composition. Screening was performed employing cell-based assays designed to test a relevant biological response for the selected indication (inhibition of cancerous cell proliferation). At the same time, the peptides were tested for their potential to inhibit the phosphorylation of the kinase substrate(s) in intact cells. The most promising peptides were chosen for further development and characterization.

\section{KinScreen: THE NEXT STEP}

The KinScreen technology developed as an outgrowth of the KinAce platform. Having developed a rapid method for constructing biologically active peptides, the next challenge was to determine potential novel biomarker targets and clinical applications for the peptides. Instead of targeting kinases with well-defined roles in specific diseases, researchers began looking at kinases whose function in signal transduction was unknown or ambiguous. The peptide inhibitors derived from a series of kinases were studied using biological assays relevant for a targeted disease. For example, to determine whether the inhibition of a specific kinase was of therapeutic value for a selected cancer, sequence-based inhibitory peptides were tested in a panel of tumor cell lines, and the inhibitory effect on cell proliferation was measured. Those peptides with the highest antiproliferative activities were further developed. Parallel studies, in which the same kinase was inhibited by alternative means [small interfering RNA (siRNA), known kinase inhibitors, in situ mutagenesis], were performed to independently verify that the kinase has a critical role in the proliferative capacity of the cancerous cells.

\section{CLINICAL APPLICATIONS}

These technologies have already been used to develop potential chemotherapeutic drug candidates and to reveal novel targets for various indications. One such candidate is KRX-123, which has been developed as a treatment for hormone refractory prostate cancer-a highly lethal disease for which therapeutic interventions are limited (3). Scientists at Keryx identified Lyn, a nonreceptor protein tyrosine kinase belonging to the Src family, as a potential target for the treatment of hormone-refractory prostate cancer. This protein had been found to have an anti-apoptotic effect on different types of tumor cells. A small-sized library of sequence-based Lyn inhibitors mimicking the $\mathrm{HJ}-\alpha \mathrm{G}$ regions was then synthesized. The peptides were tested in a cell-survival assay, and KRX-123, a nonapeptide, was found to have significant pro-apoptotic effects on the hormone refractory cell lines, DU-145 and PC3. Cell-free assays using different members of the Src protein tyrosine kinase family as targets revealed that the effect of KRX-123 was specific to Lyn. To demonstrate that the effect of KRX-123 was not the result of a nonspecific toxic effect on cancer cells, Lyn expression was reduced utilizing an RNA interference-based method (siRNA). Specific reduction in Lyn levels in DU-145 cells produced similar anti-survival responses as had KRX-123. Downstream mapping of the Lyn pathway revealed that Lyn inhibition results in decreased phosphorylation of extracellular signal-regulated kinase (ERK), a member of the mitogen-activated protein kinase (MAPK) family, which has a significant role in cell viability and growth. Finally, KRX-123 was tested in human hormone-refractory prostate cancer (HRPC) xenografts in nude mice, and the results demonstrated that KRX-123 treatment resulted in a significant reduction in tumor size and an increased percentage of apoptotic cells as compared to vehicle-treated controls.
These technologies have also been used to develop other antioncogenic, metabolic, and pro-angiogenic drug candidates $(1,4,5)$. The challenge facing the technology is that it is peptide-based, and scale-up costs for producing commercial quantities of the drug are substantial. However, significant advances in large-scale synthesis of peptides have been achieved, and even relatively long peptides, such as enfurvitide, a 36-amino acid peptide fusion inhibitor that prevents the entry of human immunodeficiency virus (HIV) into CD4+ cells, can be synthesized at the scale of 3 metric tons per year (6). Alternatively, peptides such as KRX-123 can serve as templates for generating small molecules that can be manufactured at relatively low cost.

The therapeutic potential of protein kinase inhibition, especially in the field of biomarker oncology, is now being realized (7). Novel cancer drugs such as Gleevec ${ }^{\circledR}$ (imatinib mesylate) and Iressa ${ }^{\circledR}$ (gefitinib) are already in clinical use, and their indications are being expanded. These powerful technologies facilitate rapid development of protein kinase inhibitors by using bioinformatics and structural data to develop a new class of protein-based kinase inhibitors targeting kinase-substrate interactions.

\section{COMPETING INTERESTS STATEMENT}

The authors declare no competing interests.

\section{REFERENCES}

1. Niv, M.Y., H. Rubin, J. Cohen, L. Tsirulnikov, T. Licht, A. Peretzman-Shemer E. Cna'an, A. Tartakovsky, et al. 2004. Sequence-based design of kinase inhibitors applicable for therapeutics and target identification. J. Biol. Chem. 279:1242-1255

2. Ben-Sasson, S.A., inventor. The Children's Medical Center Corp, Boston, MA and Yisum Research Development Company of the Hebrew University, Jerusalem, IL, assignees. Short peptides which selectively modulate the activity of serine-theronine kinases. 1997. U.S. patent no. 6,174,993

3. Goldenberg-Furmanov, M., I. Stein, E. Pikarsky, H. Rubin, S. Kasem, M. Wygoda, I. Weinstein, H. Reuveni, and S.A. Ben-Sasson. 2004. Lyn is a target gene for prostate cancer: sequence-based inhibition induces regression of human tumor xenografts. Cancer Res. 64:1058-1066.

4. Anis, Y., O. Leshem, H. Reuveni, I. Wexler, R. Ben Sasson, B. Yahalom, M. Laster, I. Raz, et al. 2004. Antidiabetic effect of novel modulating peptides of G-protein-coupled kinase in experimental models of diabetes. Diabetologia 47:1232-1244

5. Licht, T., L. Tsirulnikov, H. Reuveni, T. Yarnitzky, and S.A. Ben-Sasson. 2003. Induction of pro-angiogenic signaling by a synthetic peptide derived from the second intracellular loop of S1P3 (EDG3). Blood 102:2099-2107.

6. Matthews, T., M. Salgo, M. Greenberg, J. Chung, R. DeMasi, and D. Bolognesi. 2004. Enfuvirtide: the first therapy to inhibit the entry of HIV-1 into host CD4 lymphocytes. Nat. Rev. Drug Discov. 3:215-225.

7. Levitzki, A. 2003. Protein kinase inhibitors as a therapeutic modality. Acc. Chem. Res. 36:462-4699.

Address correspondence to:

Hadas Reuveni

P.O. Box 503

Har-Adar, Israel 90836

e-mail: hadas123@012.net.il 